\title{
PROPOSTA DE ESCALA DIAGRAMÁTICA PARA QUANTIFICAÇÃO DA CERCOSPORIOSE DA BETERRABA
}

\section{PROPOSAL OF SCALE DIAGRAMMATIC FOR QUANTIFICATION OF CERCOSPORA OF BEET}

\author{
Louise Larissa MAY DE MIO'1 \\ Ricardo Augusto de OLIVEIRA² \\ Andréa Mayer Veiga FLORIANI ${ }^{2}$ \\ Joselia Maria SCHUBER ${ }^{2}$ \\ Alex Sandro POLTRONIERI ${ }^{2}$ \\ Marla Alessandra de ARAUJO² \\ Renato TRATCH ${ }^{2}$
}

\begin{abstract}
RESUMO
A cercosporiose (Cercospora beticola) esta presente praticamente em todos os lugares onde se cultiva beterraba (Beta vulgaris), podendo provocar perda de rendimento de açúcar de até $40 \%$. O objetivo deste trabalho foi elaborar e validar uma escala diagramática para esta doença. Para elaboração da escala diagramática foram coletadas, aleatoriamente, 95 folhas de beterraba, na região Metropolitana de Curitiba, PR. Considerou-se a máxima e mínima proporção de área foliar lesionada e os níveis intermediários de severidade foram determinados seguindo a "Lei do estímulo de Weber e Fechner". A validação foi realizada a partir de 35 folhas de beterraba com diferentes níveis de severidade, sem e posteriormente com o auxílio da escala diagramática proposta. O uso da escala proporcionou maior acurácia e precisão das estimativas visuais, sendo indicada em estudos epidemiológicos e poderá proporcionar informações mais adequadas para estabelecer estratégias de manejo para a cercospora da beterraba.
\end{abstract}

Palavras-chave: patometria; Cercospora beticola; Beta vulgaris; severidade

\begin{abstract}
Cercospora leaf. Spot (Cercospora beticola) is present in almost all fields where beet (Beta vulgaris) is cultivated and it can cause sugar yielding loss up to $40 \%$. The aim of this work was to design and validate a diagrammatic scale for this disease. For the design of the diagrammatic scale, 95 sugar beet leaves were collected randomly in the metropolitan region of Curitiba, PR. The maximum and minimum proportions of damaged leaf area were considered and the intermediate levels of severity were determined according to "Weber and Fechner's Stimulus Law". The proposed validation of the diagrammatic scale was conducted from 35 sugar beet leaves with different levels of severity, with and without the proposed diagrammatic scale. The use of the scale provided more accuracy and precision in the visual estimates, being indicated in epidemiological studies and may provide more accurate information in order to develop management strategies for the beet's Cercospora leaf. Spot
\end{abstract}

Key-words: patometry; Cercospora beticola; Beta vulgaris; severity

'Engenheira Agrônoma, Doutora em Agronomia, Departamento de Fitotecnia e Fitossanitarismo, SCA/UFPR, Caixa Postal 2959, CEP 80035050, Curitiba/PR, e-mail: mydemio@ufpr.br. Autor para correspondência.

Engenheiro Agrônomo, Programa de Pós-graduação em Agronomia, Área de concentração em Produção Vegetal, Universidade Federal do Paraná. Rua dos Funcionários, 1540, 80035-050, Curitiba - PR. E-mail: rico@ufpr.br, andrea@casla.com.br, joseliaschuber@yahoo.com.br, alex.poltronieri@yahoo.com.br, marla.agro@bol.com.br renato.tratch@pucpr.br 


\section{INTRODUÇÃO}

A beterraba (Beta vulgaris L.) é cultivada em vários países da Europa, da América do Norte e da Ásia. Nestas regiões seu cultivo é altamente rentável e se destina à produção de açúcar e forragem (FILGUEIRA, 2000). No Brasil, a beterraba é uma das principais hortaliças cultivadas, ocupando a $13^{\text {a }}$ posição quanto ao volume de produção e apesar de ser típica de climas temperados, pode ser cultivada, praticamente, o ano todo no Estado do Paraná (MORIMOTO, 1999). É consumida, principalmente "in natura", cozida ou na forma de sucos, contudo, observa-se um crescente aumento na demanda dessa hortaliça, em indústrias de conservas e alimentos infantis (NUNES e LEITE, 2008).

Entretanto, a sua produção é limitada pela ocorrência de diversos patógenos, dentre eles o fungo Cercospora beticola Sacc., agente etiológico da cercosporiose. O fungo ataca as plantas adultas, nas quais, surgem manchas necróticas, inicialmente pequenas e rodeadas por uma pigmentação arroxeada nas faces adaxial e abaxial da folha. As manchas possuem coloração acinzentada devido à estrutura produzida pelo fungo (HERMANN, 1998).

A planta, sob ataque, diminui a sua capacidade fotossintética e repõe as folhas a partir das reservas da raiz, diminuindo assim, a produção de açúcar e a qualidade industrial. Quando em grande número, as lesões coalescem ocasionando o crestamento da folha e dá-se uma grave perda das mesmas. A planta reage emitindo novas folhas e a perda de rendimentos em açúcar pode ultrapassar os 30\% (ESPADINHA, 2007).

A quantificação de doenças é de grande importância para o desenvolvimento de medidas de controle e para os estudos epidemiológicos (BERGAMIN FILHO et al., 1995).

Para a quantificação de doenças foliares é indicado o uso da severidade (porcentagem de área foliar coberta com sintomas). Este método é aprimorado com o uso de escalas diagramáticas, pois, estas, reduzem a subjetividade da severidade estimada visualmente (GIGLIOTI e CANTERI, 1998; LEITE e AMORIM, 2002; MARTINS et al., 2004).

As escalas diagramáticas são representações ilustradas de diversas partes de plantas com sintomas em diferentes níveis de severidade (BERGAMIN FILHO e AMORIM, 1996). Atualmente, as escalas diagramáticas são as principais ferramentas de avaliação da severidade para muitas doenças. Essas devem ser de fácil uso, aplicáveis a uma grande faixa de diferentes condições, ter resultados reproduzíveis, possuir intervalo suficiente para representar todos os estágios de desenvolvimento da doença, e permitir uma avaliação imediata (BERGER, 1988). Além disto, as escalas diagramáticas devem ser validadas antes de serem propostas como um método padrão de quantificação de doenças. Considerando a inexistência de métodos padronizados para quantificação da cercosporiose da beterraba, este trabalho teve como objetivo desenvolver uma escala diagramática para a avaliação da severidade desta doença.

\section{MATERIAL E MÉTODOS}

Para elaboração da escala diagramática foram coletadas, aleatoriamente, 95 folhas de beterraba na região Metropolitana de Curitiba, PR, no ano de 2005. As folhas apresentavam diferentes níveis de áreas lesionadas pelo patógeno. Para determinação da área lesionada na lâmina foliar, utilizou-se plástico transparente, onde foi representada a área lesionada pela $C$. beticola e respectiva área foliar. Estas lâminas foram analisadas por meio do programa computacional WinRhizo, acoplado a um Scaner LA1600 (Regent Instruments Inc., Canadá), obtendo-se assim a severidade real da doença em termos percentuais. Considerou-se a máxima e mínima proporção de área foliar lesionada como sendo os valores máximo e mínimo da escala diagramática. Os níveis intermediários de severidade foram determinados seguindo a "Lei do estímulo de Weber e Fechner", de acuidade visual e resposta a incrementos logarítmicos (HORSFALL e BARRATT, 1945).

Após a definição das percentagens de área foliar lesionada e seus correspondentes níveis a serem adotados na escala, uma folha de tamanho médio, formato padrão e área conhecida, foi reproduzida seis vezes e nela representados os sintomas de cada nível. Os padrões de distribuição das lesões observados no campo foram mantidos.

A validação da escala diagramática foi realizada a partir de 35 folhas de beterraba com diferentes níveis de severidade da doença, com percentagem de severidade previamente conhecida e estas foram apresentadas a sete avaliadores. Inicialmente os avaliadores, sem experiência prévia, realizaram uma avaliação sem o auxílio da escala. Posteriormente, os mesmos avaliadores foram treinados e estimaram a severidade da doença com o uso da escala diagramática proposta.

A acurácia e a precisão das estimativas visuais de cada avaliador foram determinadas por regressão linear simples, considerando a severidade real como variável independente e a severidade estimada como variável dependente. A precisão das estimativas foi obtida pelo coeficiente de determinação da regressão $\left(\mathrm{R}^{2}\right)$ e pela variância dos erros absolutos (severidade estimada menos severidade real). Na comparação da acurácia dos dados estimados com os reais, utilizaram-se os valores da interseção (a) e da inclinação da reta (b), e na precisão os valores do coeficiente de determinação $\left(R^{2}\right)$. Foram considerados avaliadores com maior acurácia aqueles cujas estimativas proporcionaram equações de regressão linear com valores de "a" e "b" não diferindo significativamente de 0 e 1 pelo teste $t$, e 
os avaliadores com maior precisão foram considerados aqueles que tiveram ajuste do modelo linear com maiores valores de $\mathrm{R}^{2}$ (NUTTER JUNIOR e SCHULTZ, 1995).

\section{RESULTADOS E DISCUSSÃO}

A severidade de cercosporiose em beterraba na região metropolitana de Curitiba teve valor máximo de $23,61 \%$. Valores superiores não foram observados, provavelmente devido à coalescência das lesões, as quais promoveram senescência foliar; acima deste valor, não se distinguiam mais as lesões da necrose foliar. A folha no final de ciclo ou com muitas lesões fica com aspecto de dessecamento como já relatado em trabalho de outros autores (VEREIJSSEN et al., 2004). GOMES et al. (2004) em estudo para a quantificação da cercosporiose da alface, causada pelo fungo Cercospora longíssima (Cugini) Sacc. verificaram que o valor máximo de severidade da doença atingiu $68 \%$, entretanto, valores acima de $60 \%$ são raramente encontrados no campo, pois causam senescência e seca prematura da folha.

A proposta de estabelecer um sistema padronizado para orientar a avaliação da severidade de determinada doença é de grande responsabilidade, pois, caso o sistema seja deficiente, o custo de sua utilização pode ser maior do que os benefícios alcançados com seu uso (NUTTER JUNIOR e SCHULTZ, 1995; LEITE e AMORIM, 2002). No entanto, a padronização é altamente desejável, pois a uniformização da metodologia de avaliação de doenças permite comparações entre os resultados obtidos em diferentes instituições e localidades (BERGAMIN FILHO e AMORIM, 1996). A escala diagramática proposta foi composta por seis níveis de severidade, 0,$41 ; 0,97 ; 2,26 ; 5,21 ; 11,53$ e $23,61 \%$ (Figura 1 ). Para o uso desta escala, recomenda-se observar mudanças de cultivares, nutrição, região e demais condições específicas de cada cultivo.

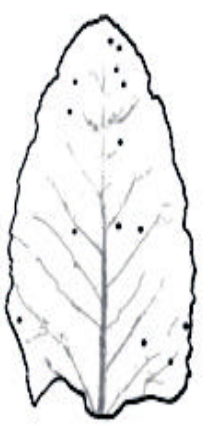

$0,41 \%$

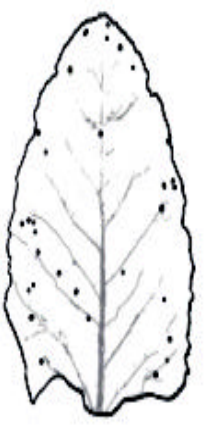

$0,97 \%$

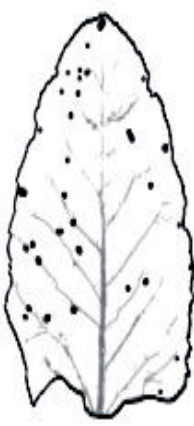

$2,26 \%$

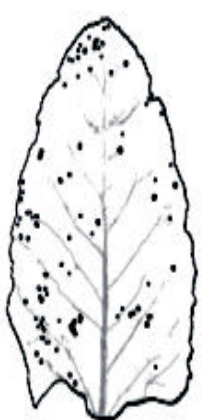

$5,21 \%$

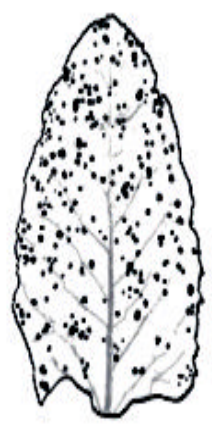

$11,53 \%$

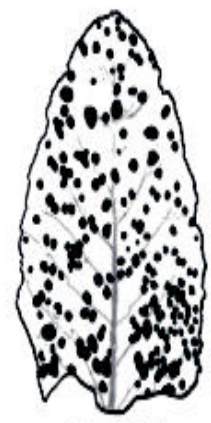

$23,61 \%$

FIGURA 1 - Escala diagramática para a avaliação da severidade da mancha de cercospora, causada pelo fungo Cercospora beticola em folhas de beterraba (Beta vulgaris). Valores em porcentagem de área foliar lesionada.

$\mathrm{Na}$ validação da escala diagramática, os avaliadores, nas condições descritas na metodologia, mostraram-se com boa acurácia, sendo que os valores estimados de severidade ficaram próximos aos valores da severidade real (Figura 2). Em cercosporiose da Alface (GOMES et al., 2004) os autores observaram valores com tendência subestimados provavelmente pela necrose pronunciada que ocorre nesta cultura a partir de $11 \%$ de severidade, e ainda neste caso a severidade máxima foi de $68 \%$. No presente trabalho apenas o avaliador quatro apresentou tendência a superestimar a doença como já observado por diversos autores em estudos com fitopatometria (HOCK et al., 1992; DIAZ et al., 2001). 

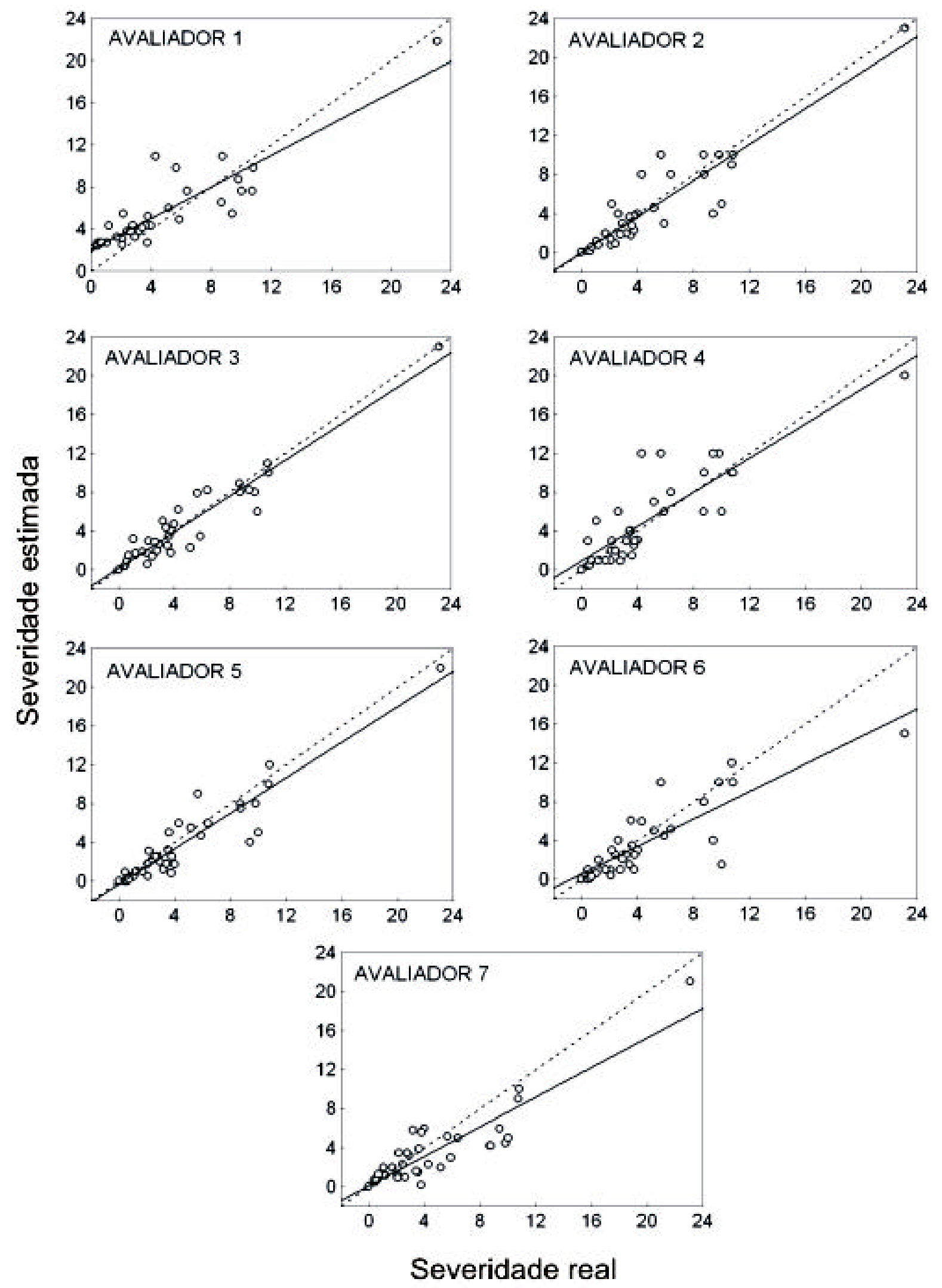

FIGURA 2 - Severidade estimada com o auxílio da escala diagramática elaborada (pontos) e linhas de regressão obtidas entre a severidade real e a estimada (linha cheia) para a mancha de cercospora em folhas de beterraba (Beta vulgaris). A linha pontilhada representa a situação ideal, com estimativas idênticas a real. 
Com a adoção da escala proposta, a totalidade dos avaliadores apresentou boa acurácia $\left(R^{2}=0,80\right)$, pois os valores de "a" não foram significativamente diferentes de zero $(0)$ e os valores de "b" não foram significativamente diferentes de um (Tabela 1).

TABELA 1 - Coeficientes lineares (a), angulares (b) e de correlação $\left(R^{2}\right)$ obtidos nas regressões entre severidade real e estimada da doença, a partir de folhas de beterraba (Beta vulgaris) com sintomas de mancha de cercospora, causados pelo fungo Cercospora beticola, com e sem o uso da escala diagramática.

\begin{tabular}{ccccccccccc}
\hline \multirow{2}{*}{ Escala } & \multirow{2}{*}{ Coeficientes } & \multicolumn{7}{c}{ Avaliadores } \\
\cline { 2 - 10 } & & 1 & 2 & 3 & 4 & 5 & 6 & 7 & Média \\
\hline \multirow{3}{*}{ Sem } & $\mathrm{a}$ & $5,84^{*}$ & $6,85^{*}$ & $4,67^{*}$ & $7,76^{*}$ & $4,49^{*}$ & $7,12^{*}$ & $6,91^{*}$ & 6,23 \\
& $\mathrm{~b}$ & 1,01 & 1,55 & 0,92 & 1,24 & 0,76 & 0,46 & 0,51 & 0,92 \\
& $\mathrm{R}^{2}$ & 0,37 & 0,32 & 0,27 & 0,20 & 0,23 & 0,15 & 0,26 & 0,26 \\
\multirow{3}{*}{ Com } & $\mathrm{a}$ & 0,13 & 0,02 & 0,19 & 0,95 & 0,32 & 0,56 & 0,09 & 0,32 \\
& $\mathrm{~b}$ & 0,68 & 0,93 & 0,92 & 0,89 & 0,91 & 0,70 & 0,75 & 0,83 \\
& $\mathrm{R}^{2}$ & 0,79 & 0,83 & 0,90 & 0,74 & 0,87 & 0,70 & 0,79 & 0,80
\end{tabular}

* Parâmetros de modelo linear que diferem significativamente de 0 e 1 para interseção (a) e inclinação (b), respectivamente.

Após a elaboração da escala a avaliação dos níveis de precisão e acurácia das devem ser analisados para determinar a qualidade das estimativas (KRANZ, 1988).

A precisão é um importante fator a ser considerado na validação de uma escala diagramática, sendo definida como a exatidão de uma operação onde há rigor ou refinamento na medida (BERGAMIN FILHO e AMORIM, 1996), e as estimativas visuais da severidade explicaram 70 a $90 \%$ da variação (R2), com média de $80 \%$ entre os avaliadores após a adoção da escala diagramática, enquanto a média sem o uso da escala era bem inferior (26\%).

Além do coeficiente de correlação, a boa precisão dos avaliadores pode ser observada com a determinação do erro absoluto ou resíduo, que corresponde à diferença entre a severidade estimada e a real (Figura 3). Os valores obtidos de resíduos foram adequados, pois ficaram entre -8 e +8 , segundo critérios adotados por programas computacionais para treinamento visual de quantificação de doenças que consideram valores entre -10 a +10 , como sendo adequados (TOMERLIN e HOWELL, 1988).

A doença causada pelo fungo $C$. beticola pode ser considerada de difícil avaliação na cultura da beterraba, uma vez que as manchas têm halo arroxeado, sendo facilmente confundidas com a coloração das nervuras das folhas, além disso, os sintomas da infecção possuem distribuição aleatória nas folhas adultas, com tamanho variando de 0,2 $0,5 \mathrm{~cm}$ de diâmetro (DUFFUS e RUPPEL, 1993). Portanto, além das técnicas adotadas para o preparo da escala, o sucesso do seu uso, medido pela precisão e acurácia na estimativa da severidade da doença, irá depender do treinamento, experiência e da percepção individual de cada avaliador (AMORIM, 1995).

A avaliação do comportamento de doenças por meio de metodologia científica e a padronização dos métodos de avaliação de doenças proporcionam maior confiabilidade no monitoramento e auxiliam em futuros estudos sobre a doença. Como JULLIATI et al. (2004) que desenvolveu uma escala para o míldio da soja, e a mesma foi utilizada por SHIGIHARA e HAMAWAKI (2005) para seleção de genótipos de soja.

A escala diagramática proposta para avaliação da cercosporiose da beterraba mostrou ser de fácil utilização, capaz de fornecer uma rápida estimativa da doença, aplicável a uma ampla gama de situações e proporcionar boa acurácia e precisão das estimativas. 

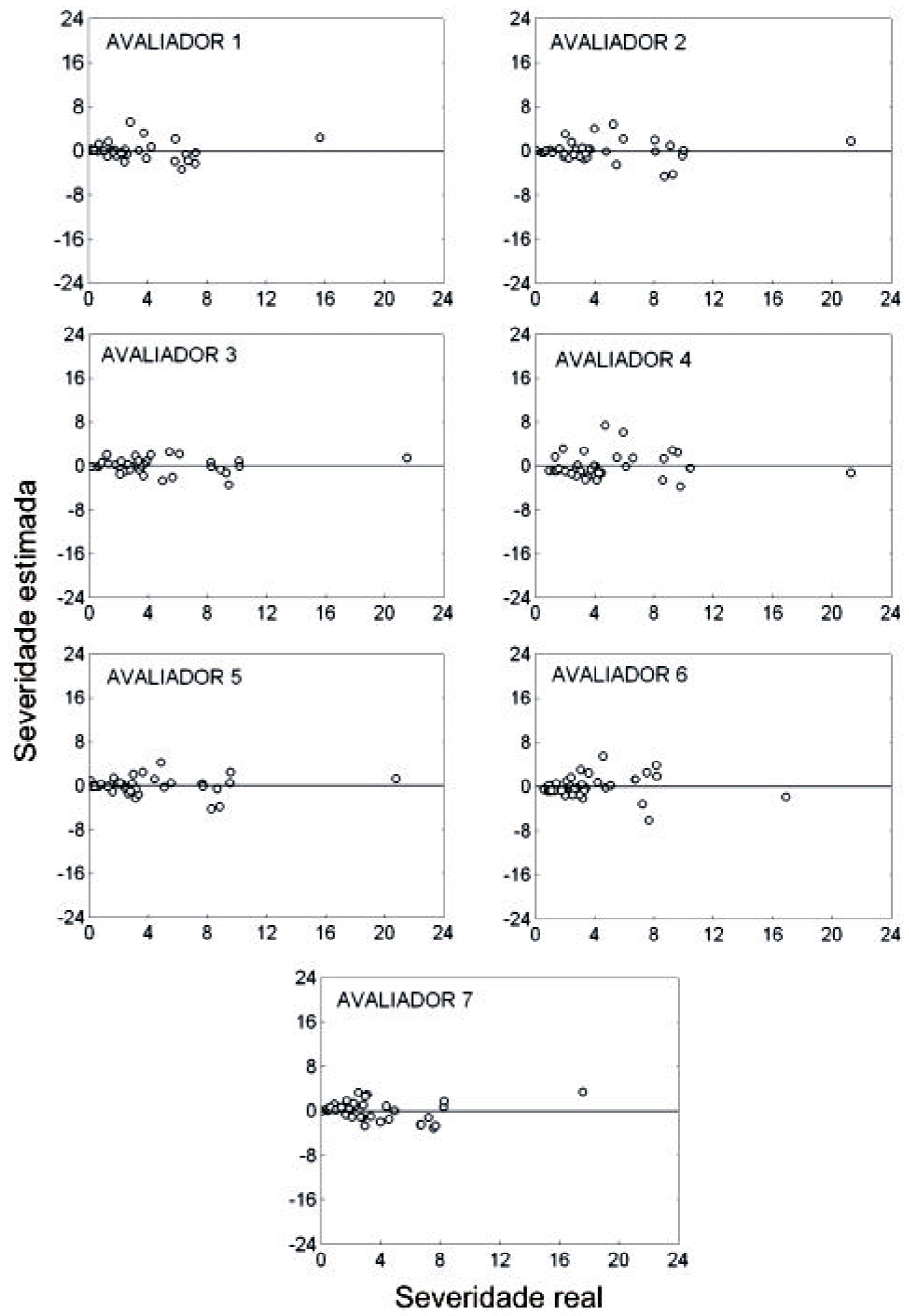

FIGURA 3 - Erros absolutos (severidade estimada menos severidade real) com o uso da escala diagramática para sete avaliadores da severidade da mancha de cercospora, causada pelo fungo Cercospora beticola em folhas de beterraba (Beta vulgaris). 


\section{CONCLUSÕES}

A utilização da escala diagramática para cercosporiose em beterraba permitiu quantificar a severidade dos sintomas de forma mais acurada e precisa.

Seu uso em estudos epidemiológicos poderá proporcionar informações mais adequadas para estabelecer estratégias de manejo para este patossistema.

\section{REFERÊNCIAS}

1. AMORIM, L. Avaliação de doenças. In: BERGAMIN FILHO, A.; KIMATI, H.; AMORIM, L. Manual de fitopatologia. 3. ed. São Paulo: Agronômica Ceres, 1995. v. 1, p. 647-671.

2. BERGAMIN FILHO, A.; KIMATI, H.; AMORIN, L. Manual de fitopatologia: princípio e conceitos. 3. ed. São Paulo: Agronômica Ceres, 1995. v. 2, 919 p.

3. BERGAMIN FILHO, A.; AMORIM, L. Doenças de plantas tropicais: epidemiologia e controle econômico. São Paulo: Agronômica Ceres, 1996. 299 p.

4. BERGER, R. D. The analysis of the effects of control measures on the development of epidemics. In: Kranz, J.; Rotem, J. (Ed.) Experimental techniques in plant disease epidemiology. Heidelberg: Springer-Verlang, 1988. p. 137-151.

5. DIAZ, C. G.; BASSANEZI, R. B.; BERGAMIN FILHO, A. Desenvolvimento e validação de uma escala diagramática para Xanthomonas axonopodis pv. phaseoli. Summa Phytopatologica, v. 27, n. 7, p. 35-39, 2001.

6. DUFFUS, J. E.; RUPPEL, E. G. Diseases. In: COOKE, D. A.; SCOTT, R. K. The Sugar beet Crop. London: Chapman \& Hall, 1993. p. 346-427.

7. ESPADINHA M. Chaves para controlar a cercosporiose na beterraba de sementeira outonal. Disponível em: <http://www.dai-sa.pt/pdfs/info_tecnica/Chaves_para_controlar_a_Cercosporiose.pdf>. Acesso em: 25/05/2007.

8. FILGUEIRA, F. A. R. Chenopodiaceae: beterraba e hortaliças herbáceas. In: FILGUEIRA, F. A. R. Novo Manual de Olericultura: agroecologia moderna e comercialização de hortaliças. Viçosa: UFV, 2000. p. 362-366.

9. GIGLIOTI, E. A.; CANTERI, M. G. Desenvolvimento de software e escala diagramática para seleção e treinamento de avaliadores do complexo broca - podridões em cana-de-açúcar. Fitopatologia Brasileira, v. 23, n. 3, p. 359-363, 1998.

10. GOMES, A. M. A.; MICHEREFF, S. J.; MARIANO, R. L. R. Elaboração e validação de escala diagramática para cercosporiose da alface. Summa Phytopathologica, v. 30, n. 1, p. 38 - 42, 2004.

11. HERMANN, O. Reconnaître les maladies foliaires de la betterave au champ. Institut Royal Belge pour l'Amérlioration de la Betterave (IRBAB/KBIVB). 1998. 20 p. Disponível em: <http://www.kbivb.be/fr/pdf/GuideMaladiesFoliaires.pdf>. Acesso em: 25/05/2007.

12. HOCK, J.; KRANZ, J.; RENFRO, B. L. Tests of standard diagrams for field use in assessing the tarspot disease complex of maize (Zea mays). Tropical Pest Management, v. 38, n. 3, p. $314-318,1992$.

13. HORSFALL, J. G.; BARRATT, R. W. An improved grading system for measuring plant disease. Phytopathology, v. 35 , p. 655, 1945

14. JULIATTI, F. C.; POLIZEL, A. C.; JULIATTI, F. C. Manejo integrado de doenças na cultura da soja. 1. ed. Uberlândia: Composer, 2004. 327 p.

15. KRANZ, J. Measuring plant disease. In: KRANZ, J.; ROTEM, J. Experimental techniques in plant disease epidemiology. Heidelberg: Springer-Verlag, 1988. p. 35-50.

16. LEITE, R. M. V. B. C.; AMORIM, L. Elaboração e validação de escala diagramática para mancha de Alternaria em girassol. Summa Phytopathologica, v. 28, n. 1, p. 14-19, 2002.

17. MARTINS, M. C.; GUERZONI, R. A.; CÂMARA, G. M. S.; MATTIAZZI, P.; LOURENÇA, S. A.; AMORIM, L. Escala diagramática para a quantificação do complexo de doenças foliares de final de ciclo em soja. Fitopatologia Brasileira, v. 29, n. 2, p. 179-184, 2004.

18. MORIMOTO, F. A oportunidade de renda e empregos com beterraba. Londrina: Emater, 1999.

19. NUNES, C.; LEITE, L., T. Cultura da beterraba. Disponível em: <http:/www3.ufla.br/ wrma luf/bth059/bth059.html>. Acesso em: 16/04/2008.

20. NUTTER JUNIOR.; F. W.; SCHULTZ, P. M. Improving the accuracy and precision of disease assessments: selection of methods and use of computer-aided training programs. Canadian Journal of Plant Pathology, v. 17, n. 1, p. 174-184, 1995.

21. SHIGIHARA, D.; HAMAWAKI, O. T. Seleção de genótipos para juvenilidade em progênies de soja. Revista Horizonte Científico, v. 4, n. 1, p. 1-26, 2005.

22. TOMERLIN, J. R.; HOWELL, T. A. Distrain: a computer program for training people to estimate disease severity on cereal leaves. Plant Disease, v. 72, n. 5, p. 455-459, 1988.

23. VEREIJSSEN, J.; SCHNEIDER, J. H. M.; TERMORSHUIZEN, A. A. J. Possible root infection of Cercospora beticola in sugar beet. European Journal of Plant Pathology, v. 110, n. 1, p. $103-106,2004$. 

